Relations industrielles

Industrial Relations

\title{
Éléments de droit social européen, par Léon-Eli Troclet, Études du Centre national de sociologie du droit social, Editions de l'Institut de Sociologie de l'Université Libre de Bruxelles, Bruxelles. 1963. 358 pages.
}

\section{Jean-Réal Cardin}

Volume 19, numéro 3, juillet 1964

URI : https://id.erudit.org/iderudit/1021287ar

DOI : https://doi.org/10.7202/1021287ar

Aller au sommaire du numéro

Éditeur(s)

Département des relations industrielles de l’Université Laval

ISSN

0034-379X (imprimé)

1703-8138 (numérique)

Découvrir la revue

Citer ce compte rendu

Cardin, J.-R. (1964). Compte rendu de [Éléments de droit social européen, par Léon-Eli Troclet, Études du Centre national de sociologie du droit social, Editions de l'Institut de Sociologie de l'Université Libre de Bruxelles, Bruxelles. 1963. 358 pages.] Relations industrielles / Industrial Relations, 19(3), 408-409. https://doi.org/10.7202/1021287ar

Tous droits réservés (C Département des relations industrielles de l’Université Laval, 1964
Ce document est protégé par la loi sur le droit d'auteur. L’utilisation des services d'Érudit (y compris la reproduction) est assujettie à sa politique d'utilisation que vous pouvez consulter en ligne.

https://apropos.erudit.org/fr/usagers/politique-dutilisation/ 
anglais après la Conquête; la proximité des Etats-Unis et de leur dynamisme irrésistibie en matière économique et industrielle, tout cela s'est conjugué en terre québécoise pour foire de notre droit un carrefour où se rencontrent et s'interpénètrent des influences souvent divergentes, peut-être appouvrissantes parfois du point de vue de ia pureté des institutions juridiques, mais souvent fécondes en initiatives créotrices, tendant vers un assouplissement nécessaire des règles de droit qu'un rigidisme trop fort rendrait inopplicable en notre contexte social actuel

II faut, à ce point, féliciter les initiateurs de cette entreprise que constitue le présent ouvrage, et en particulier le professeur Baudoin, qui depuis déjà de nombreuses années, et surtout avec son monumental traité «Le Droit civil de la province de Québec» publié en 1953, a réellement initié le mouvement actuel vers les études de droit comparé ou Québec.

II ne fout pas, évidemment, chercher dons ce recueil, des dissertotions juridiques exploront en profondeur les subtilités du droit québecois à quelque branche qu'il apportienne, mais plutôt un rappel des grands traits, des lignes de force, et des aspects les plus fondamentaux du droit québécois I'opposant ainsi aux autres grands systèmes juridiques, le français et le britannique surtout.

Pour celui qui est plus particulièrement préoccupé des problèmes socioux et de relations du travail, le présent ouvrage offre réellement matière à enrichissement et à méditation. En effet les aspects sociaux de lo réolité québécoise, et plus précisément ceux du monde industriel qui est le nôtre, sont, plus que tous les autres, affectés por le dualisme, et même la multiplicité des influences qui jouent au Québec sur les règles de droit qui les régissent.

L'étude du professeur Baudoin, par exemple, portant sur ela réception du droit étranger en droit privé québécois $\$$, étude aussi bien de sociologie du droit que de droit même, celle du professeur Louis-Philippe Taschereou sur «quelques aspects du droit judiciaire de la Province de Québec $\$$; celle de Me Phılippe Ferland sur \& l'influence du droit anglais sur la procédure civile de la Province de Québec », sont autant d'exemples où le praticien des relations du travail reconnoît et peut, grôce à eux, identifier mieux la réalité juridique dans laquelle il vit quotidiennement.

Le droit du travail, plus précisément, y est représenté por l'excellente étude de Me GuyMerrill Desaulniers, spécialiste de ce droit, étude qui peut être concise et surtout descriptive dans son ensemble n'en expose pas moins ovec maitrise les textes de base et les lignes de force de ce droit presqu'entièrement statutaire qu'est notre législation du travail.

Somme toute, un ouvrage dont on ne peut que souhoiter un prolongement dans des études subséquentes; un recueil que même les non initiés à la science juridique trouveraient profit à consulter.

Jean-Réal Cardin

Eléments de droit social européen, par LéonEli Troclet, Etudes du Centre national de sociologie du droit social, Editions de I'Institut de Sociologie de 'I'Université Libre de Bruxelles, Bruxelles. 1963. 358 pages.

II s'agit, comme l'auteur l'indique d'ailleurs dans son introduction, de la première tentative faite en vue de rassembler, dans un traité cohérent, une matière aussi incohérente et touffue que l'ensemble des textes, institutions et instruments juridiques de toutes sortes, composant ce qu'il appelle luimême le droit sccial international européen.

En effet, depuis la dernière guerre mondicle, I'Europe, afin de relever le niveau de vie des peuples qui la composent, a fait un effort remarquable d'organisation et de coopération sur tous les plans: économique, social, scientifique, commercial et même politique en dépit des nationalismes exacerbés por la guerre, des particularismes culturels, des divergences d'intérêts notionoux respectifs et des cloisonnements politiques étanches qui divisaient les peuples du vieux continent.

Une solidarité européenne jusqu'ici jamais réalisée, loin de là, est en train de se cristolliser dans une foule d'institutions qui, à leur tour, servent de treillis juridique et institutionnel empêchant un éclatement éventuel de cette «nouvelle mentalité 》 européenne, en forçant même une prise de conscience plus profonde et plus réaliste chez les diverses nations soumises à leur réseou. Aussi nous avons vu naître et se développer différents instruments de coopération qui font aujourd'hui de l'Europe, non plus le champ de bataille des empires ou des idéologies, mais davantage le centre des initiatives économiques et sociales issues d'un climat d'émulation entre les partenaires s'exprimant à l'intérieur d'une solidarité dont ils ont une conscience plus claire tous les jours. 
Bénélux, Union de l'Europe occidentale, Conseil de I'Europe, Organisation européenne de coopération économique, O.C.D.E., Marchés communs, ainsi qu'une foule d'autres institutions témoignent de cette cohésion europétnne naissante.

Mais le nombre, la diversité et la fragmentation sur le plan des moyens et des objectifs de ces instruments laissent voir oussi à quel point leur développement s'est foit à partir d'un empirisme qui laisse peu de place ou rationnel et à la logique des systèmes juridiques traditionnels. Nous sommes réellement en face d'un phénomène avant tout d'ordre sociologique, et seule une sociologie du droit, encore en gestation, peut rendre compte volablement d'un tel phénomène.

L'outeur n'o pas eu lo prétention de faire un traité de sociologie du droit ni même de présenter un traité définitif de droit social européen, puisqu'il prend la peine de bien spécifier sur la poge couverture et dans son introduction qu'il ne s'ogit tout ou plus que d'une «édition préliminaire $\gg$, loissant ou bouillonnement octuel le temps de roder les institutions et leur fonctionnement avont de songer à en tirer une analyse théorique et un système formel plus complets

Les Eléments de droit social européen constitue, en fait un premier stage vers l'élaboration d'une sociologie du droit véritable; il est un effort de collection et de présentation ordonnée des textes et des instruments existont présentement en droit social à l'échelle de l'Europe ou à tout le moins de certains pays de ce continent. $\mathrm{Ce}$ seul mérite justifie les plus grands éloges.

L'ouvroge comprend trois parties: o) les cadres institutionnels, où sont présentés, dons leurs origines, leur développement historique et leur organisotion, les principoles intstitutions étudiées; b) les instruments juridiques, où l'auteur fait la présentation et étudie les coractères et la nature des accords, conventions, résolutions, règlements etc. . qui servent d̀ mettre en oeuvre les institutions déjà décrites; c) les problèmes socioux et les solutions, qui est la partie essentielle de l'ouvrage, et où l'auteur a regroupé l'étude des institutions selon les ordres de problèmes qu'ils tendent à résoudre, afin d'en dégoger les lignes de force d'un véritable droit social européen par la comparaison des solutions apportées par les divers instruments à un même problème: les droits sociaux fondamentoux, les réfugiés et apatrides, la libre circulation des travailleurs et l'emploi, la rémunérotion, l'ossis- tance sociale et médicale, la sécurité sociole, la formation professionnelle, sont étudiés ò tour de rôle. Ainsi se dégagent les éléments d'un effort convergent entre les différentes institutions européennes tendant c̀ créer finclement un droit social cohérent ò l'échelle de ce continent.

Un traité à lire et à consulter pour tout observateur de la chose européenne à l'heure présente

\section{Jean-Réal Cardin}

A Behovioral Theory of the Firm, por Richard M. Cyert et James G. March, Prentice-Hall, Inc., Englewood Cliffs, New Jersey, 1963, 332 pages.

Les décisions constituent certainement l'activité névralgique des odministrateurs des organisations et particulièrement des entreprises. C'est ainsi que la < science administrative s'oriente de plus en plus vers l'étude du processus de prise de décision. Le présent ouvroge démontre précisément l'opport précieux du «behoviorisme », ou science du comportement, dans l'établissement d'une théorie de la décision. Les auteurs croient que pour comprendre la prise de décision économique contemporaine, il est nécessaire d'ajouter à l'étude des facteurs de marché un examen du fonctionnement interne de l'entreprise, étudier les effets de la structure de l'orgo. nisation et de la pratique conventionnelle sur le développement des buts, la formation des expectatives et l'exécution des choix.

Lo recherche qui o conduit ò ce livre se caractérise de quatre façons: 1) accent sur un petit nombre de décisions économiques clefs prises por l'entreprise; 2) développement de modèles de la firme orientés vers les processus; 3) liaison des modèles oussi près que possible ovec des observo. tions empiriques et 4 ) développement d'une théorie dont la généralité déposse les firmes spécifiques étudiées

Au cours de l'étude, l'emphase fut placée sur les processus de décision actuels. Ceci implique une description de la décision de la firme en termes d'une série spécifique d'étapes franchies pour en orriver à cette décision.

Le codre de référence fondamental d'anolyse que les outeurs proposent, comme le cadre clossique, possède deux devis majeurs d'organisation: d'abord un ensemble de catégories exhoustives de variables et ensuite un ensemble de concepts relationnels. Les outeurs prétendent pouvoir analyser le pro- 